\title{
Antithrombotic drugs in secondary stroke prevention among a community dwelling older population
}

\author{
F Landi, M Cesari, G Onder, V Zamboni, F Lattanzio, A Russo, C Barillaro, R Bernabei, \\ on behalf of the *'SILVERNET-HC Study Group
}

See end of article for authors' affiliations

.....................

Correspondence to:

Dr Francesco Landi, Istituto

di Medicina Interna e

Geriatria, Centro Medicina dell'Invecchiamento

(CEMI), Universita'

Cattolica del Sacro Cuore,

Largo Agostino Gemelli 8,

00168 Rome, Italy;

francescolandi@

rm. unicatt.it

* Listed in the

acknowledgements at the

end of the paper

\begin{abstract}
Background: Patients who suffer a cerebrovascular event are at high risk of a recurrence. Secondary prevention is crucial in reducing the burden of cerebrovascular disease.

Objective: To estimate the percentage of stroke survivors receiving antiplatelet or anticoagulant drugs and to identify factors associated with such treatment.

Design: Cross sectional retrospective cohort study.

Methods: Data were analysed from a large collaborative observational study, the Italian "silver network" home care project, which collected data (from 1997 to 2001) on patients admitted to home care programmes $(n=5372)$. Twenty two home health agencies participated in evaluating the implementation of the minimum dataset for home care (MDS-HC) instrument. For the present study, 648 individuals with a diagnosis of stroke were selected and the initial MDS-HC assessment reported.

Results: $70 \%$ of stroke survivors did not receive any antiplatelet or anticoagulant drugs $195 \%$ confidence interval (Cl), 66.5 to 73.5). Among all age categories, aspirin and ticlopidine were the two most commonly prescribed drugs. Living alone (odds ratio (OR), $0.49(95 \% \mathrm{Cl}, 0.24$ to 0.89$)$ ), dependency in activities of daily living $(0.66(0.40$ to 0.99$))$, cognitive impairment $(0.58$ (0.38 to $0.86)$ ), and low educational level $(0.58(0.34$ to 0.98$))$ were associated with a reduced likelihood of receiving secondary stroke prevention treatment. Cardiac arrhythmias, coronary artery disease, heart failure, and peripheral vascular disease were associated with the use of antiplatelet or anticoagulant treatment.

Conclusions: Negative attitudes among physicians with respect to secondary stroke prevention are prevalent and reinforce the need for increased awareness of existing data on the risks and benefits for elderly individuals. Social problems and functional impairment may be issues concerning physicians when deciding whether or not the risks of treatment exceed the benefit.
\end{abstract}

S everal randomised clinical trails have established the effectiveness of antiplatelet drugs and anticoagulant agents in the secondary prevention of stroke. ${ }^{1-6}$ For patients with a history of stroke, myocardial infarction, or other high risk vascular disease, antiplatelet agents have been shown to decrease the rate of recurrence or death from cardiovascular causes by about 30\%. ${ }^{78}$ Moreover, the American Heart Association suggests that every patient who has experienced an atherothrombotic stroke or transient ischaemic attack and has no contraindications should receive antiplatelet treatment regularly to diminish the risk of further stroke or other cardiovascular events. ${ }^{9}$

Despite much data and many recommendations, several descriptive studies have observed divergences between the best practice-as addressed by these guidelines-and the current level of treatment with antiplatelet or anticoagulant agents after cerebrovascular events. ${ }^{10-13}$ A recent observational study among nursing home residents found that $67 \%$ of stroke survivors were not receiving drug treatment for secondary stroke prevention, and that patients older than 85 years and with severe cognitive or physical impairment were even less likely to receive this kind of treatment. ${ }^{10}$

In this paper we describe a cross sectional study carried out on patients with stroke living in the community. We used the Italian "silver network" home care database to describe the patients' clinical and functional characteristics and to identify factors associated with pharmacological secondary stroke prevention.

\section{METHODS}

The study was conducted using data from the database of the Italian national home care programme called the "silver network home care project.".14 This is a population based, longitudinal, multi-linked database which comprises data collected using MDS-HC (the minimum dataset for home care) in more than 20 home health agencies in Italy, and also data on all the drugs used by each patient at the time of the MDS-HC assessment (drugs were coded using the ATC (anatomical, therapeutic and chemical) codes).

\section{MDS-HC assessment form}

The MDS-HC ${ }^{15}$ contains over 350 data elements, including sociodemographic variables, numerous clinical items about both physical and cognitive status, and all clinical diagnoses. The MDS-HC also includes information about an extensive array of signs, symptoms, syndromes, and treatments provided. ${ }^{16}$ Various different multi-item summary scales are embedded in the MDS-HC-measuring, for example, physical function (activities of daily living (ADL); instrumental activities of daily living (IADL), ${ }^{17}$ and cognitive status (cognitive performance scale (CPS)). ${ }^{17}{ }^{18}$ The MDS items have excellent inter-rater and test-retest reliability when completed by

Abbreviations: $A D L$, activities of daily living; $C P S$, cognitive performance scale; IADL, instrumental activities of daily living; MDS-HC, minimum dataset for home care 
Table 1 Sociodemographic, functional, and clinical variables in patients with stroke*

\begin{tabular}{|c|c|}
\hline Characteristics & Total $(n=648)$ \\
\hline Age (years) (mean (SD)) & $78.7(9.6)$ \\
\hline Female & $362(56)$ \\
\hline \multicolumn{2}{|l|}{ Marital status } \\
\hline Married & $316(48)$ \\
\hline Widowed & 277 (43) \\
\hline Never married & $55(9)$ \\
\hline Living alone & $95(15)$ \\
\hline \multicolumn{2}{|l|}{ Education } \\
\hline Low (0-5 years) & 118 (18) \\
\hline Medium-high ( $>5$ years) & $530(82)$ \\
\hline ADL score (mean (SD)) & $5.5(2.1)$ \\
\hline IADL score (mean (SD)) & $5.6(2.1)$ \\
\hline CPS score (mean (SD)) & $2.7(1.8)$ \\
\hline Impaired cognitive performance $†$ & $356(55)$ \\
\hline \multicolumn{2}{|l|}{ Cardiovascular diseases } \\
\hline Hypertension & $290(45)$ \\
\hline Coronary artery disease & $131(20)$ \\
\hline Heart failure & $113(17)$ \\
\hline Peripheral vascular disease & $152(23)$ \\
\hline Cardiac arrhythmias & 126 (19) \\
\hline \multicolumn{2}{|l|}{ Other diseases } \\
\hline Diabetes mellitus & $133(20)$ \\
\hline Depression & $316(48)$ \\
\hline Dementia & 124 (19) \\
\hline \multicolumn{2}{|l|}{ Preventive treatment } \\
\hline Aspirin & $96(14)$ \\
\hline Dipyridamole & $5(0)$ \\
\hline Ticlopidine & $67(10)$ \\
\hline Warfarin & $27(4)$ \\
\hline \multicolumn{2}{|c|}{$\begin{array}{l}\text { *Data are given as } n(\%) \text { unless otherwise indicated. } \\
\text { †CPS score } \geqslant 2 \text { or more. } \\
\text { ADL, activities of daily living (range } 0-7 \text {, a higher number indicates greater impairment); CPS, cognitive } \\
\text { performance scale (range } 0-6 \text {, a higher number indicates greater impairment); IADL, instrumental activities of } \\
\text { daily living (range } 0-7 \text {, a higher number indicates greater impairment). }\end{array}$} \\
\hline
\end{tabular}

nurses performing their usual assessment duties (average weighted $\kappa=0.8) .{ }^{18} 19$

\section{Study sample}

The study population consisted of all patients admitted to home care programmes in 22 home health agencies from 1997 to 2001 who participated in the national silver network project $(\mathrm{n}=5372)$. The intended study sample was all those with a diagnosis of stroke reported on initial MDS-HC assessment $(n=699)$. From this we excluded any whose drug treatment was not known accurately $(n=51)$, leaving a final sample of 648. The MDS-HC diagnosis of stroke is based on physician's interpretation of the patient's medical history as presented by physical examination, the medical record, and hospital discharge documentation.

\section{Drug classification}

Home care staff recorded the ATC code for up to 18 drugs taken within the seven days preceding the MDS-HC assessment. The MDS-HC drug inventory has been shown to be both consistent and reliable. ${ }^{17}$ We considered that secondary pharmacological prevention of stroke was reflected in standing orders for the antiplatelet agents aspirin (B01AC06), dipyridamole (B01AC07), and ticlopidine (B01AC05), and the anticoagulant

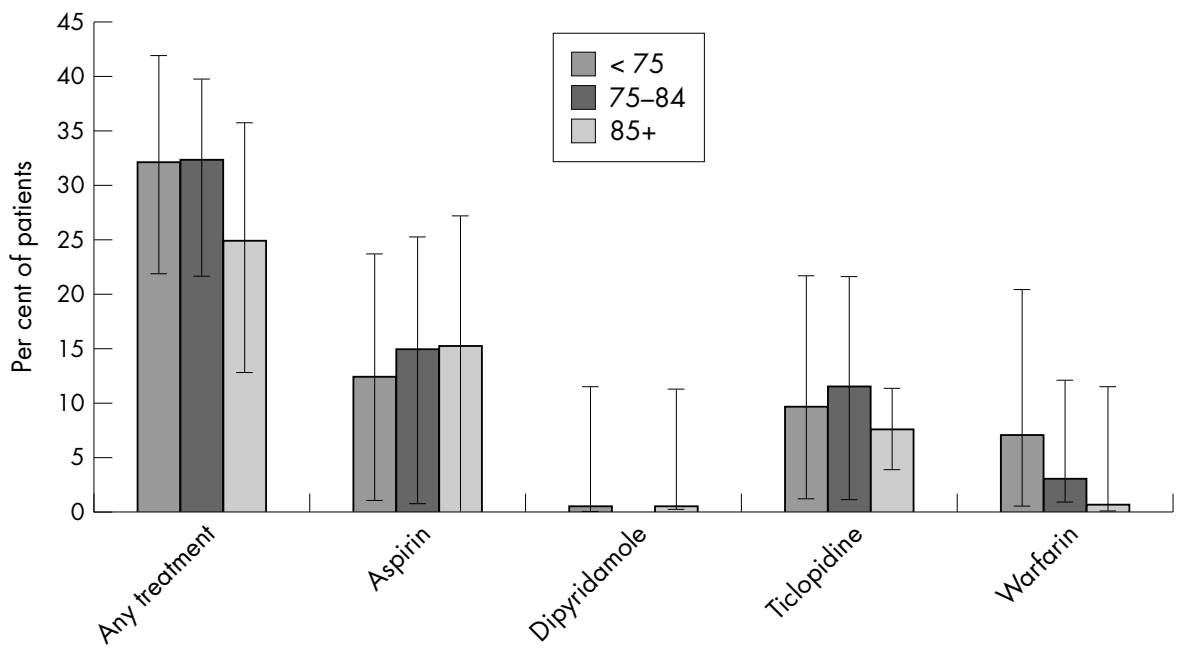

Figure 1 Pharmacological treatment of patients with stroke, stratified by age categories (prevalences and $95 \%$ confidence intervals). 
Table 2 Main characteristics of participants and odd ratios for any antiplatelet or anticoagulant treatment

\begin{tabular}{|c|c|c|c|c|c|}
\hline Variable & $\begin{array}{l}\text { Treated } \\
(n=195)\end{array}$ & $\begin{array}{l}\text { Not treated } \\
(n=453)\end{array}$ & $\%$ Treated & $\begin{array}{l}\text { Univariate odds ratio } \\
(95 \% \mathrm{Cl})\end{array}$ & $\begin{array}{l}\text { Adjusted model* odds } \\
\text { ratio }(95 \% \mathrm{Cl})\end{array}$ \\
\hline \multicolumn{6}{|l|}{ Age (years) } \\
\hline 65 to 74 & 67 & 141 & 32 & 1.0 (reference) & 1.0 (reference) \\
\hline 75 to 84 & 79 & 165 & 32 & $1.0(0.67$ to 1.49$)$ & $1.13(0.73$ to 1.74$)$ \\
\hline$\geqslant 85$ & 49 & 147 & 25 & $0.70(0.45$ to 1.08$)$ & $0.97(0.59$ to 1.61$)$ \\
\hline \multicolumn{6}{|l|}{ Sex } \\
\hline Male & 84 & 202 & 29 & 1.0 (reference) & 1.0 (reference) \\
\hline Female & 111 & 251 & 31 & $1.06(0.75$ to 1.49$)$ & 1.15 (0.79 to 1.65$)$ \\
\hline Living alone & 21 & 74 & 22 & 0.61 (0.36 to 1.03$)$ & $0.49(0.24$ to 0.89$)$ \\
\hline \multicolumn{6}{|l|}{ Education } \\
\hline Medium-high ( $>5$ years) & 172 & 358 & 32 & 1.0 (reference) & 1.0 (reference) \\
\hline Low (0-5 years) & 23 & 95 & 19 & $0.50(0.30$ to 0.82$)$ & $0.58(0.34$ to 0.98$)$ \\
\hline Compromised ADL function $†$ & 151 & 394 & 28 & 0.51 (0.33 to 0.79$)$ & $0.66(0.40$ to 0.99$)$ \\
\hline Impaired cognitive performance & 84 & 272 & 23 & 0.49 (0.35 to 0.70$)$ & $0.58(0.38$ to 0.86$)$ \\
\hline \multicolumn{6}{|l|}{ Cardiovascular diseases } \\
\hline Hypertension & 96 & 194 & 33 & $1.29(0.92$ to 1.81$)$ & 1.27 (0.85 to 1.90$)$ \\
\hline Coronary artery diseases & 52 & 79 & 40 & $1.71(1.15$ to 2.56$)$ & 1.73 (1.08 to 2.77 ) \\
\hline Heart failure & 41 & 72 & 36 & $1.40(0.91$ to 2.15$)$ & 1.53 (0.92 to 2.56$)$ \\
\hline Peripheral vascular disease & 56 & 96 & 37 & 1.49 (1.02 to 2.19 ) & 1.63 (0.99 to 2.67$)$ \\
\hline Cardiac arrhythmias & 52 & 74 & 41 & 1.86 (2.24 to 2.78$)$ & 1.96 (1.20 to 3.18$)$ \\
\hline \multicolumn{6}{|l|}{ Other diseases } \\
\hline Diabetes mellitus & 39 & 94 & 29 & 0.95 (0.62 to 1.45$)$ & 0.93 (0.58 to 1.47$)$ \\
\hline Depression & 91 & 225 & 29 & 0.88 (0.63 to 1.24$)$ & 0.93 (0.64 to 1.33 ) \\
\hline Dementia & 33 & 91 & 27 & $0.81 \quad(0.52$ to 1.25$)$ & $1.01(0.58$ to 1.47$)$ \\
\hline \multicolumn{6}{|c|}{$\begin{array}{l}\text { *Adjusted simultaneously for all the variables listed. } \\
\dagger \text { ADL score of at least } 2 \text { (range } 0-7 \text {, a higher number indicates greater impairment). } \\
\text { fCognitive performance scale (CPS) score of } 2 \text { or more (range } 0-6 \text {, a higher number indicates greater impairment). } \\
\text { ADL, activities of daily living; Cl, confidence interval. }\end{array}$} \\
\hline
\end{tabular}

warfarin (B01AA03). Clopidogrel was not considered because it only became available in Italy in 2000, after the study protocol and data collection were completed. No patient in the sample population was taking acenocumarol (B01AA07) at the assessment time.

\section{Statistical analysis}

Data were analysed to obtain descriptive statistics. Continuous variables are presented as mean (SD). Age trends for the use of antiplatelet or anticoagulant agents were analysed using the $\chi^{2}$ test for trend. A probability $(p)$ value of $<0.05$ was chosen for statistical significance.

To identify predictors of secondary stroke preventive treatment, we selected a sample of patients with a diagnosis of stroke and ran a logistic regression model using treatment with any antiplatelet or anticoagulant agent as the dependent variable. We adjusted our model for age, sex, and any potential confounding variables (diabetes, hypertension, cardiac arrhythmia, coronary artery disease, congestive heart failure, peripheral vascular disease, and social, functional, and cognitive status). The IADL score was excluded from the multivariate analysis to limit the confounding effect of colinearity with ADL. We did not consider economic factors in our analyses because the Italian National Health Plan gives universal coverage including the provision of drugs. From the final model, we derived odds ratios (OR) and the corresponding 95\% confidence intervals (CI). Statistical analyses were undertaken using SPSS software.

\section{RESULTS}

Main characteristics of the study sample population are shown in table 1. Patients were white, predominately female $(56 \%)$, with a mean (SD) age of 78.7 (9.6) years. Sixty eight per cent of the individuals were aged 75 years or more. Overall, patients had a moderate to severe impairment in basic and instrumental activities of daily living; similarly, cognitive function was compromised in a large number of patients $(55 \%$ had a CPS score more than 2, indicating moderate to severe cognitive impairment). On average, the sample had a relatively good level of formal education. Widows and widowers formed $43 \%$ of the sample, and $15 \%$ lived alone without any available informal care.

Seventy per cent of individuals with a diagnosis of stroke had not received any antiplatelet drugs or warfarin (95\% CI, 66.5 to 73.5). Among all age categories, aspirin and ticlopidine were the two most commonly prescribed drugs. Figure 1 shows the relation between age and the secondary stroke prevention treatment. With increasing age, a lower proportion of patients with stroke received any antiplatelet or anticoagulant drugs ( $32 \%, 32 \%$, and $25 \%$ of patients in the 65 to 74 years, 75 to 84 years, and 85 years + groups, respectively; $p=0.06$ for trend). This trend was significantly evident only for warfarin treatment. However, only 3\% of patients aged 75 years and older received this drug, compared with $8 \%$ of patients aged 65 to 74 years $(\mathrm{p}=0.005)$.

Table 2 shows predictive factors for secondary preventive treatment among patients with stroke. Living alone (OR, 0.49 ( $95 \%$ CI, 0.24 to 0.89$)$ ), dependency in activities of daily living (0.66 (0.40 to 0.99$))$, cognitive impairment (0.58 (0.38 to $0.86))$, and low educational level $(0.58(0.34$ to 0.98$))$ were associated with a reduced likelihood of receiving secondary stroke prevention treatment. Conversely, cardiac arrhythmia, coronary artery disease, heart failure, and peripheral vascular disease were associated with receiving anticoagulant or antiplatelet treatment.

\section{DISCUSSION}

Stroke is one of the leading causes of morbidity, disability, dependency, and mortality in the USA and in Western 
countries. Patients who have experienced a cerebrovascular event are at high risk of a recurrence, and secondary prevention is the most important intervention to decrease the burden of cerebrovascular disease. Various different treatments have been shown to be effective in lowering the risk of recurrent stroke. Several antiplatelet drugs are useful in the secondary prevention of cerebral ischaemic events and in reducing stroke and myocardial infarction. ${ }^{1-620}$ The American Heart Association guidelines for secondary stroke prevention recommend the use of warfarin in all patients with atrial fibrillation who have no contraindications to taking the drug. ${ }^{21}$

A few studies have examined the prevalence of undertreatment of cerebrovascular disease in elderly stroke survivors living in the community. ${ }^{11-13}$ In particular, Petty and colleagues ${ }^{11}$ studied a community dwelling population with a previous history of cerebrovascular accidents and found that $30 \%$ of their sample were not treated with any anticoagulant or antiplatelet agents. There are few data about factors influencing the choice of a specific treatment for secondary prevention in patients with a recent stroke or transient ischaemic attack.

Our results in the present study show that only $30 \%$ of individuals who suffered a cerebrovascular event received any secondary preventive treatment. Patients aged 85 years or older seemed less likely to receive drugs for secondary stroke prevention than younger patients, though this trend was significant only for warfarin. Subjects with physical or cognitive impairment were also less likely to receive preventive drug treatment than the non-impaired population. In addition, the absence of any informal caregiver and a low educational level were associated with a decreased likelihood of treatment within our sample.

The observation that older, functionally impaired and demented persons were at greater risk of receiving no treatment is of special concern, as shown in previous studies. ${ }^{12}{ }^{122-24}$ In this respect, demographic and social problems and functional impairment may be linked to the physician's decision about whether or not the potential risks of treatment exceed any possible benefit. In these particular situations, physicians may postpone the prescription of antiplatelet drugs or warfarin because of a perceived inability to monitor high risk patients effectively..$^{25}$ Moreover, Kutner and colleagues ${ }^{26}$ found that general practitioners were unwilling to prescribe antiplatelet or anticoagulant drugs for their elderly patients living in the community.

Non-treatment is not the same as undertreatment. ${ }^{27}$ Contraindications such as gastrointestinal bleeding, peptic ulcer disease, patient's or family's wishes, and treatment intolerance can also be expected to play an important role in a physician's decision to postpone or start any type of secondary preventive treatment. In the light of these considerations, there are some limitations to our study that need to be recognised. First, we have no detailed clinical information about specific contraindications to warfarin or antiplatelet treatment. It is possible that differences in the frequency of contraindications might account for some of the demographic association (for example, contraindications certainly increase in very old people). Second, the MDS-HC stroke diagnosis does not distinguish between haemorrhagic and ischaemic strokes. However, despite these limitations, it is implausible that contraindications can explain the large gap between the recommended and observed rates of treatment that we documented in our study. It is also highly improbable that the $70 \%$ of patients who were not receiving any antiplatelet or anticoagulant drugs had suffered haemorrhagic strokes.

A more critical consideration is that indecision about secondary preventive treatment is not limited to potential risks in frail elderly patients. There is also uncertainty about the possible benefits. ${ }^{28}$ The most important evidence on antiplatelet or anticoagulant drugs after cerebrovascular events is based on "non-disabling" ischaemic stroke. Scientific evidence about the risks and benefits of secondary stroke pre- ventive treatment is much more limited for patients with severe physical disability or cognitive impairment.

\section{Conclusions}

The negative attitudes of general practitioners over pharmacological treatment for stroke prevention strengthen the need to increase their knowledge of existing data on risks and benefits for frail elderly subjects. However, further research into the outcomes of such preventive treatments is warranted. More clearly defined guidelines suggesting indications for specific therapies are needed, especially among frail and functionally impaired older individuals who have suffered a cerebrovascular event.

\section{ACKNOWLEDGEMENTS}

The Silvernet-HC Study Group is composed of the following:

Steering Committee: R Bernabei, P U Carbonin, M P Ruffilli; Coordination: F Landi, F Lattanzio; Writing Panel: G Gambassi, A Russo, M Cesari, L Manigrasso, F Pagano, C Gobbi, G Onder, A Sgadari; Participants: the list has been published in Aging Clin Exp Res 1999;11:262-72 and in Anziani Oggi 2000;3-4:152.

This study was supported by a grant from the "Progetto Finalizzato Invecchiamento" of the National Research Council.

Authors' affiliations

F Landi, V Zamboni, A Russo, C Barillaro, R Bernabei, Department of Gerontology, Catholic University of Sacred Heart, Rome, Italy M Cesari, G Onder, Sticht Center on Aging, Wake Forest

University-Baptist Medical Center, Winston Salem, North Carolina, USA F Lattanzio, Pfizer Italiana SpA, Rome, Italy

Competing interests: none declared

\section{REFERENCES}

1 Caprie Steering Committee. A randomized, blinded, trial of clopidogrel versus aspirin in patients at risk of ischemic events (CAPRIE). Lancet 1996;348: 1329-38

2 Diener HC, Cunha L, Forbes C, et al. European Stroke Prevention Study. II. Dipyridamole and acetylsalicylic acid in the secondary prevention of stroke. J Neurol Sci 1996;143:1-13.

3 Gent M, Easton J, Hachinski V, et al. The Canadian American ticlopidine study (CATS) in thromboembolic stroke. Lancet 1989;i:1215-20.

4 SALT Collaborative Group. Swedish Aspirin Low-Dose Trial (SALT) of $75 \mathrm{mg}$ of aspirin as secondary prophylaxis after cerebrovascular ischemic events. Lancet 1991:338:1345-9.

5 Forbes CD. Secondary stroke prevention with low-dose aspirin, sustained release dipyridamole alone and in combination. ESPS Investigators European Stroke Prevention Study. Thromb Res 1998;92:S1-6.

6 EAFT Study Group. Secondary prevention in non-rheumatic atrial fibrillation after transient ischaemic attack or minor stroke. Lancet 1993;342: 1255-61.

7 Gaspoz JM, Coxson PG, Goldman PA, et al. Cost effectiveness of aspirin, clopidogrel, or both for secondary prevention of coronary heart disease. N Engl J Med 2002:346:1800-6.

8 Antiplatelet Trialists' Collaboration. Collaborative overview of randomised trials of antiplatelet therapy. I. Prevention of death, myocardial infarction, and stroke by prolonged antiplatelet therapy in various categories of patients. BM 1994;308:8/1-106.

9 Albers GW, Easton JD, Sacco RL, et al. Antithrombotic and thrombolytic therapy for ischemic Stroke. Chest 1998; 1 14:683-98S.

10 Quilliam BJ, Lapane KL. Clinical correlates and drug treatment of residents whit stroke in long-term care. Stroke 2001;32:1385-93.

11 Petty GW, Brown RD, Whisnant JP, et al. Frequency of major complications of aspirin, warfarin, and intravenous heparin for secondary stroke prevention: a population-based study. Ann Intern Med 1999; 130:14-22

12 Moroney JT, Tseng C-L, Paik MC, et al. Treatment for the secondary prevention of stroke in older patients: the influence of dementia status. $J$ Am Geriatr Soc 1999:47:824-9.

13 Rockwood D, Ebly E, Hachinsky V, et al. Presence and treatment of vascular risk factors in patients with vascular cognitive impairment. Arch Neurol 1997;54:33-9.

14 Landi F, Lattanzio F, Gambassi G, et al. A model for integrated home care of frail older patients: the Silver Network Project. Aging Clin Exp Res 1999;11:262-72.

15 Morris JN, Fries BE, Steel K, et al. Comprehensive clinical assessment in community setting: applicability of the MDS-HC. J Am Geriatr Soc 1997;45: 1017-24.

16 Morris JN, Fries BE, Bernabei R, et al. RAl - Home Care assessment manual. Washington, DC: InterRAl Corporation, 1996. 
17 Landi F, Tua E, Onder G, et al. The minimum data set for home care: a valid instrument to assess frail older people living in community. Med Care 2000;38: $1184-90$

18 Hartmaier SL, Sloane PD, Guess HA, et al. Validation of the minimum data set cognitive performance scale: agreement with the mini-mental state examination. J Gerontol 1995:50:M128-33.

19 Hawes C, Morris JN, Phillips CD, et al. Reliability estimates of the minimum data set (MDS) for nursing home resident assessment and care screening. Gerontologist 1995;2:172-8.

20 Dippel DWJ. The results of CAPRIE, IST and CAST. Thromb Res 1998:92:S13-16.

21 Wolf PA, Clagett GP, Easton JD, et al. Preventing ischemic stroke in patients with prior stroke and transient ischemic attack: a statement for healthcare professionals from the stroke council of the American Heart Association. Stroke 1999:30:1991-4.

22 Landi F, Onder G, Zuccalà G, et al. Non-scientific prescription in demented older people living in the community: an Italian perspective. Clin Drug Invest 2001;21:157-60
23 Landi F, Gambassi G, Lapane KL, et al. Comorbidity and drug use in cognitively impaired elderly living in long-term care. Dement Geriatr Cogn Disord 1998;9:347-56.

24 Landi $F$, Onder $G$, Cesari $M$, et al. Pain management in frail, community-living elderly patients. Arch Intern Med 2001;161: 2721-4.

25 Monette J, Gurwitz JH, Rochon PA, et al. Physician attitudes concerning warfarin for stroke prevention in atrial fibrillation: results of a survey of long-term care practitioners. J Am Geriatr Soc 1997;45: 1060-65.

26 Kutner M Nixon G, Silverstone F. Physicians' attitudes toward oral anticoagulants and antiplatelet agents for stroke prevention in elderly patients whit atrial fibrillation. Arch Intern Med 1991;151:1950-3.

27 Leibson C. Management of stroke in long-term care [editorial]. Stroke 2001:32:1392-3.

28 Gage BF, Fihn SD, White RH. Warfarin therapy for an octogenarian who has atrial fibrillation. Ann Intern Med 2001;134:465-74.

\title{
NEUROLOGICAL STAMP
}

\author{
August von Wassermann (1866-1925)
}

W

assermann was a German physician and bacteriologist who was educated at the Universities of Erlangen, Vienna, Munich, and Strasbourg. He graduated in 1888. From 1890 he was a student of Robert Koch at the Institute of Infectious Diseases in Berlin and in 1907 became head of the department of therapeutics and serum research. In 1913 he moved to the Kaiser Wilhelm Institute. Here he was director of experimental therapeutics until his death 12 years later. Wassermann is best remembered for the Wassermann test or reaction, which he developed in association with the German dermatologist Albert Neisser. The Wassermann test was the predecessor of today's Venereal Disease Research Laboratory (VDRL) test for the diagnosis of infection with syphilis. Wassermann also developed a treatment for diphtheria and vaccinations for cholera, tetanus, and typhoid fever. Alhough not portrayed on a postage stamp, Wassermann has been portrayed on a German postmark.

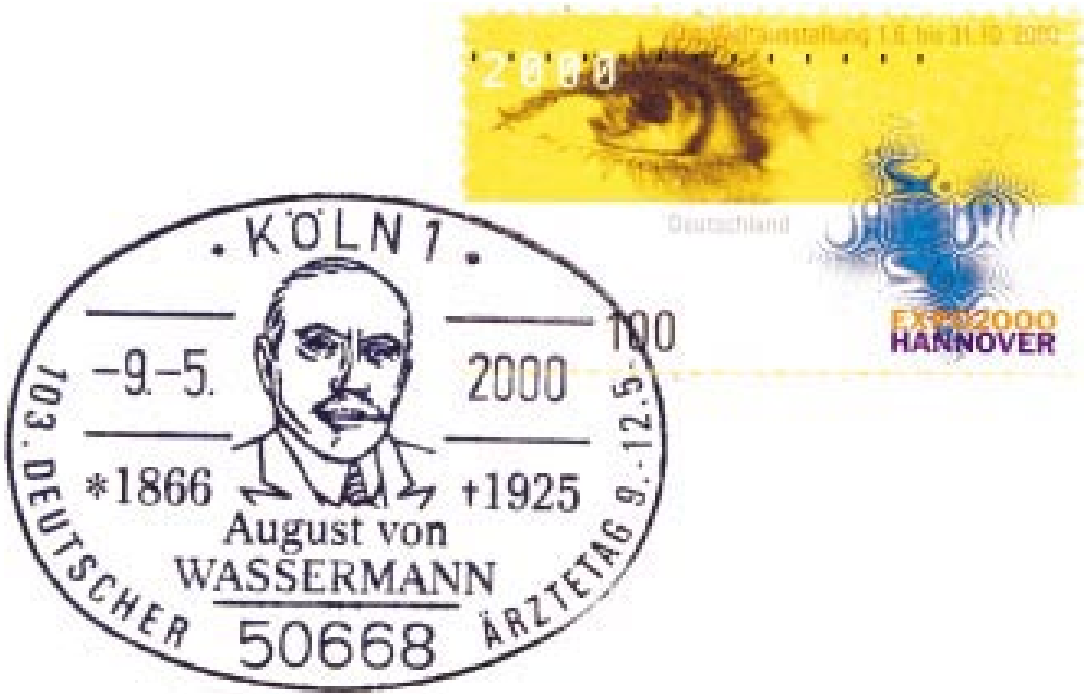

L F Haas 